\title{
2 N An \\ Reaction of vinylbromides with alkynes using ppm amounts of Pd as catalyst
}

\author{
André F. P. Biajoli and Adriano L. Monteiro \\ Laboratory of Molecular Catalysis, Instituto de Química - UFRGS. \\ Av. Bento Gonçalves 9500, 91501-970, CP 15003, Porto Alegre, RS, Brazil. \\ *corresponding author: pivatobiajoli@yahoo.com
}

Keywords: Sonogashira, 1,3-enynes, low Pd levels

\section{INTRODUCTION}

The 1,3-enyne is an important core structure often found in many naturally occurring and biologically active compounds. In addition, 1,3-enynes are also very useful synthetic intermediates. The Pdcatalyzed Sonogashira reaction is one of the most powerful methods for the synthesis of such compounds. Pd complexes containing phosphine ligands associated with copper(I) iodide are the most widely used catalysts for this reaction. ${ }^{1}$ In the past several years, some protocols using copper catalysts have been developed. ${ }^{2}$ Recently, we have shown that a catalytic system based on $\mathrm{Cul} / 1,10$ phenanthroline efficiently promotes the coupling between phenols or pyrazole with (E)bromostilbene. ${ }^{3}$ Here we present our first results about the coupling of vinyl bromides with alkynes.

\section{RESULTS AND DISCUSSION}

We choose as reaction model the cross-coupling of (E)-bromostilbene with phenylacetylene (Figure 1).
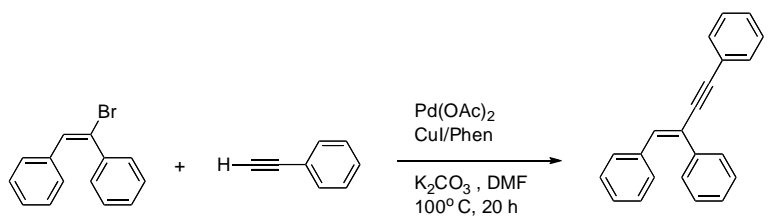

Figure 1. Coupling of (E)-bromostilbene with phenylacetylene

We have performed a screening of bases, solvents, temperature and ligand using $\mathrm{Cul}$ as catalyst. Despite an initial observation of the cross-coupling product, the results obtained indicated that contamination with $\mathrm{Pd}$ was responsible for the reaction. In fact, the dramatic effect of ppb levels of $\mathrm{Pd}$ on the Cu-catalyzed Sonogashira coupling of aryl halides has been recently pointed out. Therefore, we decided to investigate the effect of low palladium concentrations in the reaction (Figure 1 and Table 1). After an optimization, we obtained most complete conversion on the 1,3-enyne product using only 40 ppm of Pd and 5 mol\% of Cul (entry 3). Lower yields were obtained when lower amounts of $\mathrm{Cu}$ (entries 12) or $\mathrm{Pd}$ (entries 4-6) were used. It is important to mention that the desired product was obtained in moderate yield using only $0,1 \mathrm{ppm}$ of $\mathrm{Pd}$ with a TON of 196,000 (entry 6). On the other hand, a $9 \%$ yield was observed when $\mathrm{Pd}(\mathrm{OAc})_{2}$ was not added (entry 7).

Table 1. Effect of Pd and Cu on the cross-coupling of (E)bromostilbene with phenylacetylene ${ }^{a}$

\begin{tabular}{|c|c|c|c|}
\hline Entry & $\begin{array}{c}\text { Ratio } \\
\text { Substrate/Pd }(\mathrm{OAc})_{2}\end{array}$ & $\begin{array}{c}\text { Cul } \\
(\mathrm{mol} \%)\end{array}$ & $\begin{array}{l}\text { Yield }^{b} \\
(\%)\end{array}$ \\
\hline 1 & 1,000 & 1 & 57 \\
\hline 2 & 1,000 & 2.5 & 70 \\
\hline 3 & 1,000 & 5 & 99 \\
\hline 4 & 4,000 & 5 & 86 \\
\hline 5 & 40,000 & 5 & 72 \\
\hline 6 & 400,000 & 5 & 48 \\
\hline $7^{c}$ & - & 5 & 9 \\
\hline action & conditions: (E)-bro & Dene & Cul $\quad \mathrm{mm}$ \\
\hline
\end{tabular}

\section{CONCLUSION}

The Sonogashira coupling reaction of vinyl bromides was efficiently performed using low amounts of Pd. Further studies concerning the application of this reaction and the role of $\mathrm{Pd}$ and $\mathrm{Cu}$ are in progress.

\section{ACKNOWLEDGEMENTS}

We thank CNPq, PRONEX-FAPERGS, and INCTCatálise for partial financial support. We also thank CNPq for scholarship (A.F.P. Biajoli).

\section{REFERENCES}

1 See for example: Feuerstein, M.; Chahen, L.; Douce, H.; aMaurice Santelli, M.; Tetrahedron. 2006, 62, 112.

${ }^{2}$ See for example: Saha, D; Chatterjee, T; Mukherjee, M; Ranu, B.C.; J. Org. Chem. 2012, 77, 9379.

${ }^{3}$ Limberger,J.; Leal, B.C.; Back, D.F.; Dupont, J.; Monteiro, A.L.; Adv Synth. Catal. 2012, 354, 1429 